\title{
Vacancy induced half-metallicity in half-Heusler semiconductors
}

\author{
Zhiyong Zhu, Yingchun Cheng, and Udo Schwingenschlögl* \\ Physical Sciences and Engineering Division, KAUST, Thuwal 23955-6900, Kingdom of Saudi Arabia
}

(Received 31 August 2011; published 28 September 2011)

\begin{abstract}
First-principles calculations are performed to investigate the effect of vacancies on the electronic structure and magnetic properties of the two prototypical half-Heusler semiconductors NiTiSn and CoTiSb. The spin degeneracy of the host materials is broken for all types of isolated vacancies under consideration, except for Ni-deficient NiTiSn. A half-metallic character is identified in Sn-deficient NiTiSn and Co/Ti/Sb-deficient CoTiSb. We can explain our findings by introducing an extending Slater-Pauling rule for systems with defects. A ferromagnetic ordering of the local moments due to double exchange appears to be likely.
\end{abstract}

DOI: 10.1103/PhysRevB.84.113201

PACS number(s): 71.20.Be, 75.50.Pp, 85.75.-d

The half-Heusler alloys form a family of materials with AgAsMg-type $C 1_{b}$ structure and stoichiometric composition $X Y Z$, where $X$ and $Y$ are usually transition metals and $Z$ is an $s p$ element. If the number of valence electrons of the halfHeusler alloy $X Y Z$ is 18 , a gap is opened at the Fermi level by strong $d-d$ hybridization between the $X$ and the $Y$ atoms. ${ }^{1-3}$ A nonmagnetic semiconducting ground state survives even in the presence of transition metals with open $d$ shells. Recently, great interest has focused on the half-Heusler semiconductors with 18 valence electrons, which comprise promising candidates for high-performance thermoelectric materials, ${ }^{4-7}$ halfmetallic dilute magnetic semiconductors, ${ }^{8-12}$ and the recently discovered three-dimensional topological insulators. ${ }^{13-15}$

One important feature of the 18-electron half-Heusler semiconductors is the coexistence of open $d$ shells and a nonmagnetic ground state. As a direct result, the spin degeneracy is sensitive to defects, disorder, and dopants. This fact has been exploited to develop new types of dilute magnetic semiconductors. For example, replacement of Ti by other $3 d$ transition elements in semiconducting NiTiSn and CoTiSb results in a new type of dilute magnetic semiconductor with half-metallic properties. ${ }^{8-12}$ Particularly, the high Curie temperature $(>700 \mathrm{~K})$ and small lattice mismatch in $\mathrm{Fe}$ substituted CoTiSb makes the compound to a serious candidate for applications in magnetoelectronics and spintronics. ${ }^{10,11}$ Vacancies are point defects that occur inherently in all crystalline materials, with equilibrium concentrations of up to $0.1 \% .^{16,17}$ Vacancy induced magnetism in metal oxides has been a topic of intensive research in recent years. ${ }^{18-21}$ In the 18electron half-Heusler semiconductors, vacancies are likewise expected to heavily influence the electronic structure and magnetic behavior. Experimental investigation with atomic resolution is possible by state-of-the-art positron annihilation spectroscopy. ${ }^{22,23}$

In this paper, the effect of vacancies on the prototypical 18-electron half-Heulser semiconductors NiTiSn and CoTiSb is analyzed by means of first-principles calculations. Local magnetic moments are induced in the nonmagnetic host around all the addressed vacancies except for Ni deficiency in NiTiSn. In addition, a half-metallic character is reflected by the electronic structures of Sn-deficient NiTiSn as well as $\mathrm{Co} / \mathrm{Ti} / \mathrm{Sb}$-deficient $\mathrm{CoTiSb}$. It will be necessary to extend the Slater-Pauling rule to fully explain the observed vacancy effects. The tendency of the vacancy induced local magnetic moments toward a long-range ferromagnetic ordering will be discussed in terms of the exchange mechanism and the possibility of percolation of the exchange interaction.

The characteristic crystal structure of half-Heusler alloys with space group $F \overline{4} 3 m$ can be described in terms of three interpenetrating fcc lattices with atomic positions $X(0,0,0)$, $Y(0.25,0.25,0.25)$, and $Z(0.75,0.75,0.75)$ (see Fig. 1). Full potential linearized augmented plane-wave calculations are performed, making use of the WIEN2K package, ${ }^{24}$ which treats magnetic systems with a high accuracy. ${ }^{25,26}$ We use $2 \times 2 \times 2$ supercells containing $32 X Y Z$ primitive cells to simulate the dilute limit of the magnetic system. The valence states of the involved elements are $\mathrm{Ni} 3 p^{6} 3 d^{10} 4 s^{2} 4 p^{6}$, Ti $3 s^{2} 3 p^{6} 3 d^{10} 4 s^{2} 4 p^{6}$, and Sn $4 p^{6} 4 d^{10} 5 s^{2} 5 p^{6} 5 d^{10}$. Moreover, we set $R_{\mathrm{mt}}=2.1$ a.u., $R_{\mathrm{mt}} K_{\max }=7$, and $l_{\max }=10$, and employ the identical $6 \times 6 \times 6 k$ mesh in all our calculations. The atoms in the vicinity of the vacancy are fully relaxed with a force tolerance of only $0.5 \mathrm{mRy} /$ Bohr. Pulay corrections are implemented into the force's calculations.

Both the local density approximation ${ }^{27}$ (LDA) and generalized gradient approximation ${ }^{28}$ (GGA) are employed to calculate the basic properties of pure NiTiSn and CoTiSb. They yield the same nonmagnetic semiconducting ground state. Beyond, the calculated band gaps of $0.5 \mathrm{eV}$ for NiTiSn and $1.0 \mathrm{eV}$ for CoTiSb are identical. The theoretical GGA lattice constants of $5.997 \AA$ for NiTiSn and $5.884 \AA$ for CoTiSb agree very well with the experimental values of $5.941 \AA$ for NiTiSn and $5.884 \AA$ for $\mathrm{CoTiSb}{ }^{1}$ In contrast, the LDA predicts much too small values of $5.806 \AA$ for NiTiSn and $5.784 \AA$ for CoTiSb. Therefore, the GGA will be used in the following calculations.

Spin-polarized calculations are performed to evaluate the effect of vacancies on the magnetic properties. While a Ni vacancy has hardly any effect on the magnetic state of the NiTiSn host, spin polarization is induced next to $\mathrm{Ti}$ and $\mathrm{Sn}$ vacancies. Without structural relaxation, the corresponding magnetic moments both are equal to $2.00 \mu_{B}$. After the relaxation of the atomic forces around the defect, the magnetic moment induced by the Ti vacancy is slightly reduced to $1.93 \mu_{B}$, indicating a sensitivity of the magnetism to the structural changes. On the other hand, the magnetic moments induced by $\mathrm{Co}$, Ti, and $\mathrm{Sb}$ vacancies in CoTiSb are 1.00, 2.00, and $1.00 \mu_{B}$, respectively, unaffected by the structural relaxation.

In Fig. 2, we next study the electronic density of states (DOS) obtained from the spin-polarized calculations for pure 


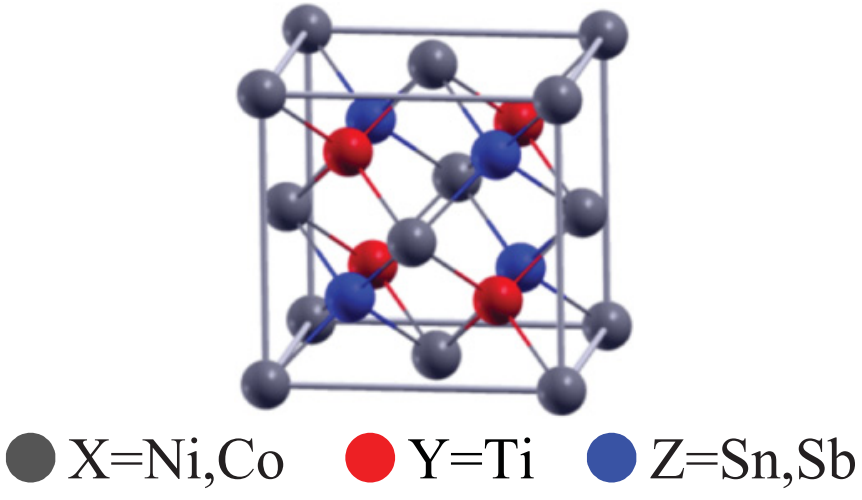

FIG. 1. (Color online) Unit cell of a half-Heusler alloy $X Y Z$, comprising four formula units.

NiTiSn and NiTiSn with $\mathrm{Ni}$, Ti, and Sn vacancies after the structural relaxation. Considering the semiconducting state of the NiTiSn host, no smearing is applied to the DOS in order to avoid artificial metallic states at the Fermi energy $\left(E_{F}\right)$. Nonmagnetic ground states are found for both pure NiTiSn and $\mathrm{Ni}$-deficient NiTiSn. In contrast, spin polarization is induced in Ti/Sn-deficient NiTiSn. Before the relaxation, these two systems show a half-metallic nature, which agrees with the integer magnetic moments. After the relaxation, Sn-deficient NiTiSn retains its half-metallicity, while Ti-deficient NiTiSn develops a finite amount of minority spin states at $E_{F}$. This explains the deviation of the magnetic moment from $2 \mu_{B}$. The DOS calculated for pure CoTiSb and CoTiSb with Co, Ti, and $\mathrm{Sb}$ vacancies after the structural relaxation is shown in Fig. 3. In contrast to NiTiSn, all three types of vacancies in CoTiSb cause a spin polarization. Furthermore, the half-metallic character is robust in all cases, as reflected by integer magnetic moments.

For both NiTiSn and CoTiSb, the defect states lie inside the semiconducting band gap of the host. In the case of NiTiSn, the width of the Ti-defect band is larger than that of the Sn-defect band, while in the case of CoTiSb, the Co-defect band is much broader than the Ti- and Sb-defect bands. Considering the energetical position of the defect states with respect to the valence- and conduction-band edges of the host, different defect band widths are related to different degrees of hybridization between the defect and host states.

To explain the vacancy induced half-metallic behavior and the integer magnetic moment, we have to extend the SlaterPauling rule, which applies to many full- and half-Heusler alloys. ${ }^{3,29-31}$ The rule states that the total magnetic moment per primitive cell $\left(M_{t}\right)$ scales with the total number of valence electrons $\left(Z_{t}\right)$ as $M_{t}=Z_{t}-N_{b}$, where the material-dependent parameter $N_{b}$ is equal to 18 and 24 for half- and full-Heusler alloys, respectively. This value is the number of occupied bands (accounting for spin degeneracy) below a gap $E_{g}$ just below the Fermi energy $\left(E_{F}\right)$ in the nonpolarized band structure. ${ }^{3,31}$ The main idea is that excess electrons, which can not be accommodated in the $N_{b}$ bands below $E_{g}$, become completely spin polarized. As a result, a half-metallic state with an integer magnetic moment is formed. In the half-Heusler alloys (see the discussion of $\mathrm{NiMnSb}$ as a prototypical example in Ref. 3), the $s p$ element $Z$ introduces a deep-lying $s$ band.

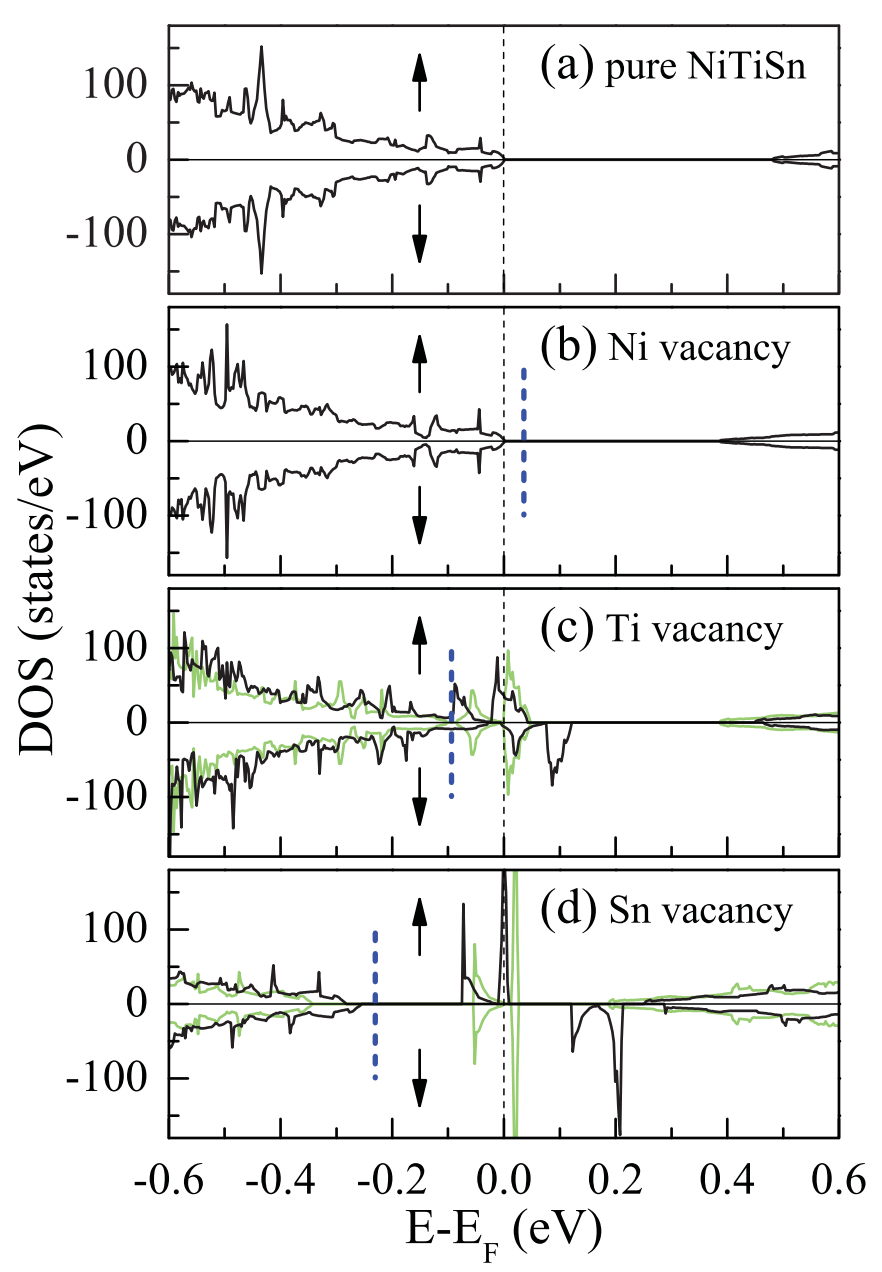

FIG. 2. (Color online) Electronic density of states (DOS) as obtained for (a) the pure NiTiSn $2 \times 2 \times 2$ supercell and for supercells with (b) Ni, (c) Ti, and (d) Sn deficiency. The dark and light lines indicate spin-polarized and nonpolarized calculations, respectively. Majority and minority spin states are labeled $\uparrow$ and $\downarrow$, respectively. Short vertical dashed lines mark the value $E_{g}$ of the extended Slater-Pauling model.

In addition, hybridization with the $d t_{2 g}$ states shifts the three $p$ bands below the center of the $d$ states. The $s$ and $p$ bands accommodate a total of eight electrons per primitive cell. Hybridization between the low-energy $d$ states of the high-valence transition-metal atom $X$ and the high-energy $d$ states of the low-valence transition-metal atom $Y$ now leads to bonding and antibonding states with energy gap at $E_{g}$. The five bonding $d$ bands accommodate 10 electrons in total. Therefore, we obtain $N_{b}=18$ and a nonmagnetic semiconducting ground state for half-Heusler alloys.

In our present supercell calculations with vacancy, the total magnetic moment of the supercell that comprises $n$ primitive cells can be written as $M_{T}=n Z_{t}-Z_{v}-N_{B}$, where $Z_{v}$ denotes the number of valence electrons of the removed atom and $N_{B}$ has the same meaning as $N_{b}$ but refers to the supercell. For both NiTiSn and CoTiSb, we have $n=32$ and $Z_{t}=18$. Also, $Z_{v}=9,10,4,4$, and 5 for $\mathrm{Co}, \mathrm{Ni}, \mathrm{Ti}, \mathrm{Sn}$, and $\mathrm{Sb}$, respectively. For all vacancy types in NiTiSn and CoTiSb, $N_{B}$ is obtained by a nonpolarized calculation. The resulting DOS is included in Figs. 2 and 3, which also indicate the position 


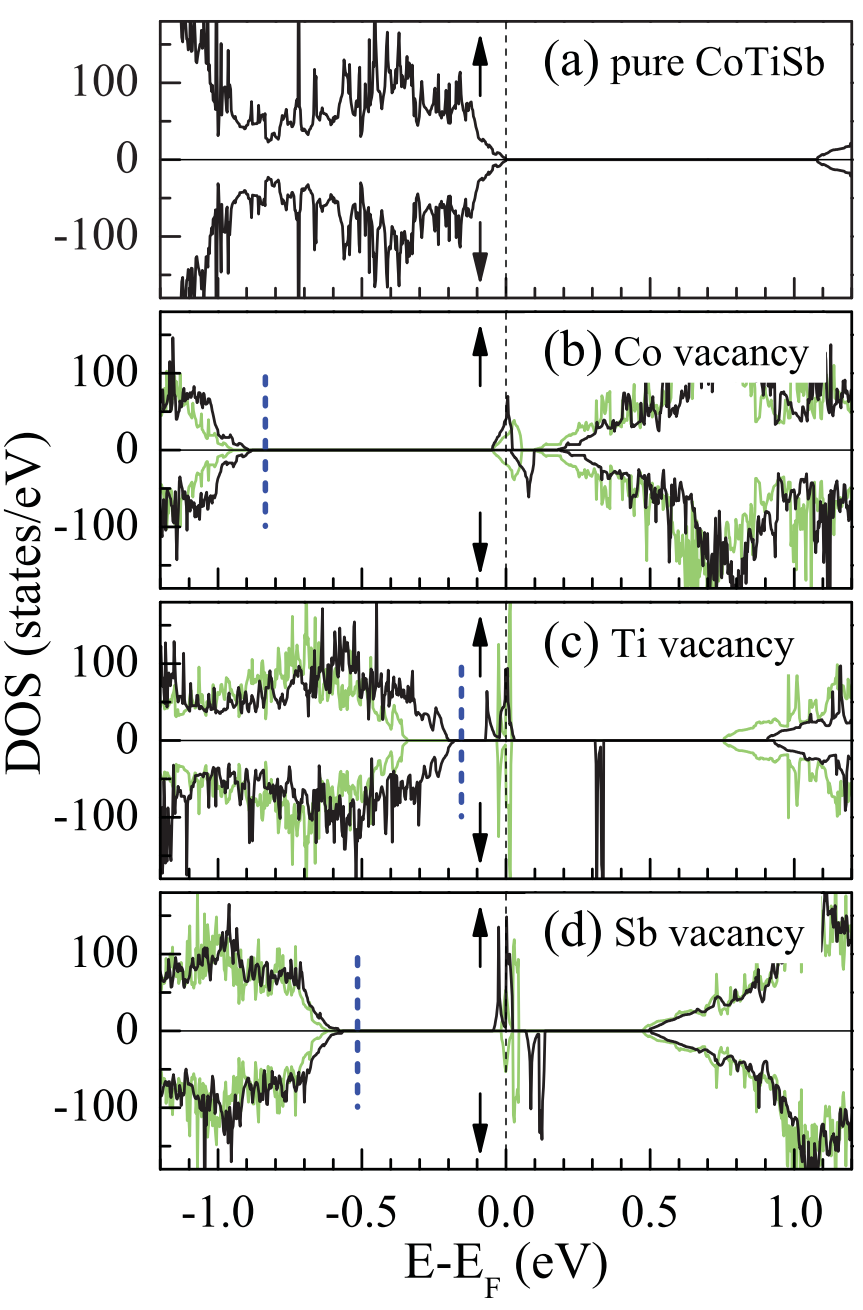

FIG. 3. (Color online) Electronic density of states (DOS) as obtained for (a) the pure CoTiSb $2 \times 2 \times 2$ supercell and for supercells with (b) $\mathrm{Co}$, (c) $\mathrm{Ti}$, and (d) Sb deficiency. The dark and light lines indicate spin-polarized and nonpolarized calculations, respectively. Majority and minority spin states are labeled $\uparrow$ and $\downarrow$, respectively. Short vertical dashed lines mark the value $E_{g}$ of the extended Slater-Pauling model.

of $E_{g}$ by short vertical dashed lines. We find $N_{B}=566$ for Co and $\mathrm{Ni}$ vacancies and $N_{B}=570$ for $\mathrm{Ti}, \mathrm{Sn}$, and $\mathrm{Sb}$ vacancies. The extended Slater-Pauling rule now leads to $M_{T}=0,2$, and 2 for $\mathrm{Ni}, \mathrm{Ti}$, and $\mathrm{Sn}$ defects in NiTiSn, respectively. For $\mathrm{Co}$, Ti, and $\mathrm{Sb}$ defects in CoTiSb, we obtain $M_{T}=1$, 2 , and 1 , respectively. These findings perfectly agree with the first-principles magnetic moments, except for the case of Ti-deficient NiTiSn, where the structural relaxation destroys the half-metallic state.

Apparently, the parameter $N_{B}$ depends only on the location of a vacancy but not on the atomic species. This indicates that the extended Slater-Pauling rule can also be deduced from the modifications of the hybridization between the removed atom and its surrounding. For an $X$ vacancy, the 10 bonding $d$ bands below $E_{g}$, which belong to the low-energy $d$ states of the high-valence transition-metal atom $X, 2,3,12$ disappear. Since the total number of valence electrons is reduced by $Z_{v}, 10-Z_{v}$ bands shift above $E_{g}$ into the semiconducting gap and become fully spin polarized. The situation is quite different for a $Y$ vacancy. Even though the $d$ states of the low-valence transition-metal atom $Y$ contribute mainly to the antibonding bands above the semiconducting energy gap of the host, removal of $d-d$ hybridization with the $X$ bands [mainly, $t_{2 g}$ (Refs. 2,3,12)] raises the latter in energy. Consequently, $E_{g}$ lies between the $t_{2 g}$ and $e_{g}$ bands, $N_{B}$ is reduced by 6 , and $6-Z_{v}$ bands shift into the semiconducting gap and become fully spin polarized. Finally, in the case of a $Z$ vacancy, the $s$ and $p$ bands below $E_{g}$ are lost. However, a new $s$ band is created below $E_{g}{ }^{3}$ As a result, $6-Z_{v}$ excess bands shift into the semiconducting gap and become fully spin polarized. Simple arithmetic then gives us the same result as deduced from the band-structure calculation. In addition, the orbital hybridization argument allows us to derive a concise form of the extended Slater-Pauling rule: $M_{T}=\Delta N_{B}-Z_{v}$, where $\Delta N_{B}$ depends only on the site of the vacancy with values of 10 for $X$ and 6 for $Y$ and $Z$ defects.

We stress that the effect of vacancies on the electronic structure and magnetism resembles isostructural doping with atoms that have a different number of valence electrons. Substitution of $\mathrm{Sb}$ by $\mathrm{Sn}$ in $\mathrm{CoTiSb}$ and of $\mathrm{Ni}$ by $\mathrm{Co}$ in NiTiSn causes a crossover from a semiconductor to a magnetic metal. ${ }^{1}$ Beyond, substitution of Ti by Mn in NiTiSn gives rise not only to room-temperature ferromagnetism, but also to half-metallicity. ${ }^{9}$ Half-metallic ferromagnetism is also encountered when Ti is replaced by $\mathrm{Cr} / \mathrm{Mn} / \mathrm{Fe}$ in CoTiSb, with the Curie temperature of the Fe-doped alloy far above room temperature. ${ }^{10,11}$ The main difference between substitution and deficiency concerns only the creation of the excess electrons. Substitution of Ti by $\mathrm{Cr} / \mathrm{Mn} / \mathrm{Fe}$ in NiTiSn and CoTiSb yields excess charge that can not be accommodated in $N_{B}$ bands. It becomes fully spin polarized and a half-metallic state arises. If vacancies are introduced, the valence charge is reduced. However, this reduction is overcompensated by a lower $N_{B}$, and we have an effective excess charge.

Finally, we ask the question as to whether ferromagnetic or antiferromagnetic ordering prevails between the vacancy induced local magnetic moments. Three mechanisms are relevant in dilute magnetic systems. ${ }^{32}$ Zener's doubleexchange $\mathrm{e}^{33,34}$ as well as $p-d$ exchange $^{35}$ mechanisms favor a ferromagnetic coupling, while superexchange ${ }^{36}$ usually yields an antiferromagnetic coupling. According to Figs. 2(c) and 2(d) and 3(b)-3(d), the spin-polarized defect states in the semiconducting gap of the host are only partially filled. This eliminates both superexchange and $p-d$ exchange because the former is suppressed when $E_{F}$ lies in the defect bands and the latter needs the valence bands of the host to be sandwiched between the majority and minority spin defect $d$ bands. ${ }^{32}$ As a matter of fact, the nearly half-filled defect bands and an exchange interaction exceeding the band width (see Figs. 2 and 3) favor strongly double exchange and, thus, ferromagnetism in the present systems.

Another important factor for the formation of a longrange order in dilute magnets is the spatial extension of the exchange interaction. If the interaction is localized and can not percolate the crystal, the magnetism will be strongly suppressed in the dilute limit. For NiTiSn and CoTiSb with defects, such a localized nature in fact may be expected. On the other hand, when Ti is substituted by Fe in CoTiSb, ${ }^{11}$ the half-metallic character is similar to our defect systems and 
a remarkable ferromagnetism is found experimentally even for a $\mathrm{Fe}$ concentration as low as $0.3 \%$, pointing to strong percolation of the exchange interaction. Because the vacancy concentration can be as high as $0.1 \%$ in our systems, ${ }^{16,17}$ i.e., comparable to the above Fe concentration, long-range ferromagnetism is expected to be induced by the vacancies.

In conclusion, the effects of vacancies in the prototypical 18-electron half-Heusler semiconductors NiTiSn and CoTiSb on the electronic structures and magnetic properties have been analyzed by full potential first-principles calculations. We obtain local magnetic moments induced into the nonmagnetic host materials for all types of isolated vacancies in NiTiSn and CoTiSb, except for the Ni vacancy in NiTiSn. In addition, half-metallicity is found for Sn-deficient NiTiSn and $\mathrm{Co} / \mathrm{Ti} / \mathrm{Sb}$-deficient CoTiSb. The Slater-Pauling rule of bulk half- and full-Heusler alloys has been extended to systems with defects to explain the findings. It is to be expected that this extended rule can also provide additional insights into the vacancy induced magnetism in metal oxides. ${ }^{18-21}$ Moreover, our data indicate that the long-range ferromagnetic ordering in NiTiSn and CoTiSb is induced by the double-exchange mechanism. *udo.schwingenschlogl@kaust.edu.sa

${ }^{1}$ J. Tobola, J. Pierre, S. Kaprzyk, R. V. Skolozdra, and M. A. Kouacou, J. Phys. Condens. Matter 10, 1013 (1998).

${ }^{2}$ B. R. K. Nanda and I. Dasgupta, J. Phys. Condens. Matter 15, 7307 (2003).

${ }^{3}$ I. Galanakis, P. H. Dederichs, and N. Papanikolaou, Phys. Rev. B 66, 134428 (2002).

${ }^{4}$ C. Uher, J. Yang, S. Hu, D. T. Morelli, and G. P. Meisner, Phys. Rev. B 59, 8615 (1999).

${ }^{5}$ J. Barth, B. Balke, G. H. Fecher, H. Stryhanyuk, A. Gloskovskii, S. Naghavi, and C. Felser, J. Phys. D: Appl. Phys. 42, 185401 (2009).

${ }^{6}$ S. Sakurada and N. Shutoh, Appl. Phys. Lett. 86, 082105 (2005).

${ }^{7}$ L. Chaput, J. Tobola, P. Pécheur, and H. Scherrer, Phys. Rev. B 73, 045121 (2006).

${ }^{8}$ S. Ouardi, G. H. Fecher, B. Balke, X. Kozina, G. Stryganyuk, C. Felser, S. Lowitzer, D. Kodderitzsch, H. Ebert, and E. Ikenaga, Phys. Rev. B 82, 085108 (2010).

${ }^{9}$ B. Sanyal, O. Eriksson, K. G. Suresh, I. Dasgupta, A. K. Nigam, and P. Nordblad, Appl. Phys. Lett. 89, 212502 (2006).

${ }^{10}$ B. Balke, G. H. Fecher, A. Gloskovskii, J. Barth, K. Kroth, C. Felser, R. Robert, and A. Weidenkaff, Phys. Rev. B 77, 045209 (2008).

${ }^{11}$ K. Kroth, B. Balke, G. H. Fecher, and H. J. Lin, Appl. Phys. Lett. 89, 202509 (2006).

${ }^{12}$ B. R. K. Nanda and I. Dasgupta, J. Phys. Condens. Matter 17, 5037 (2005).

${ }^{13}$ D. Xiao, Y. Yao, W. Feng, J. Wen, W. Zhu, X.-Q. Chen, G. M. Stocks, and Z. Zhang, Phys. Rev. Lett. 105, 096404 (2010).

${ }^{14}$ S. Chadov, X. L. Qi, J. Kübler, G. H. Fecher, C. Felser, and S. C. Zhang, Nat. Mater. 9, 541 (2010).

${ }^{15}$ H. Lin, L. A. Wray, Y. Q. Xia, S. Y. Xu, S. Jia, R. J. Cava, A. Bansil, and M. Z. Hasan, Nat. Mater. 9, 546 (2010).

${ }^{16}$ R. W. Siegel, J. Nucl. Mater. 69, 117 (1978).

${ }^{17}$ M. G. Meere, J. R. King, and T. G. Rogers, Int. J. Numer. Model. El. 3, 99 (1990)
${ }^{18}$ I. S. Elfimov, S. Yunoki, and G. A. Sawatzky, Phys. Rev. Lett. 89, 216403 (2002).

${ }^{19}$ C. Das Pemmaraju and S. Sanvito, Phys. Rev. Lett. 94, 217205 (2005).

${ }^{20}$ G. Rahman, V. M. García-Suárez, and S. C. Hong, Phys. Rev. B 78, 184404 (2008).

${ }^{21}$ H. Peng, J. Li, S.-S. Li, and J.-B. Xia, Phys. Rev. B 79, 092411 (2009).

${ }^{22}$ Y. Fan, A. Kushima, S. Yip, and B. Yildiz, Phys. Rev. Lett. 106, 125501 (2011).

${ }^{23}$ S. Kilpeläinen, F. Tuomisto, J. Slotte, J. L. Hansen, and A. N. Larsen, Phys. Rev. B 83, 094115 (2011).

${ }^{24}$ P. Blaha, K. Schwarz, G. K. H. Madsen, D. Kvasnicka, and L. Luitz, WIEN2K, 2001.

${ }^{25}$ U. Schwingenschlögl, C. Schuster, and R. Frésard, Europhys. Lett. 81, 27002 (2009); Ann. Phys. (Berlin) 18, 107 (2009).

${ }^{26}$ U. Schwingenschlögl and C. Schuster, Phys. Rev. Lett. 99, 237206 (2007); Eur. Phys. J. B 55, 43 (2007).

${ }^{27}$ J. P. Perdew and Y. Wang, Phys. Rev. B 45, 13244 (1992).

${ }^{28}$ J. P. Perdew, K. Burke, and M. Ernzerhof, Phys. Rev. Lett. 77, 3865 (1996).

${ }^{29}$ J. Kübler, Phys. B \& C (Amsterdam) 127, 257 (1984).

${ }^{30}$ D. Jung, H. J. Koo, and M. H. Whangbo, J. Mol. Struct.: THEOCHEM 527, 113 (2000).

${ }^{31}$ I. Galanakis, P. H. Dederichs, and N. Papanikolaou, Phys. Rev. B 66, 174429 (2002).

${ }^{32}$ K. Sato, L. Bergqvist, J. Kudrnovský, P. H. Dederichs, O. Eriksson, I. Turek, B. Sanyal, G. Bouzerar, H. Katayama-Yoshida, V. A. Dinh, T. Fukushima, H. Kizaki, and R. Zeller, Rev. Mod. Phys. 82, 1633 (2010).

${ }^{33}$ C. Zener, Phys. Rev. 82, 403 (1951).

${ }^{34}$ H. Akai, Phys. Rev. Lett. 81, 3002 (1998).

${ }^{35}$ C. Zener, Phys. Rev. 81, 440 (1951).

${ }^{36}$ H. A. Kramers, Physica (Amsterdam) 1, 182 (1934). 